\title{
Heatstroke-induced late-onset neurological deficits in mice caused by Purkinje cell degeneration, demyelination, and synaptic impairment at cerebellum
}

Kazuyuki Miyamoto ( $\nabla$ k-miyamoto@med.showa-u.ac.jp )

Showa University

Motoyasu Nakamura

Showa University

Hirokazu Ohtaki

Showa University

Keisuke Suzuki

Showa University

Hiroki Yamaga

Showa University

Kaoru Yanagisawa

Showa University

Atsuo Maeda

Showa University

Masaharu Yagi

Showa University

Munetaka Hayashi

Showa University

Kazuho Honda

Showa University

Kenji Dohi

Showa University

\section{Research Article}

Keywords: Heatstroke, Cerebellum, Late-onset neurological deficits, Demyelination, Purkinje cells degeneration, Synaptic impairment

Posted Date: February 28th, 2022 
DOI: https://doi.org/10.21203/rs.3.rs-1341515/v1

License: (c) (1) This work is licensed under a Creative Commons Attribution 4.0 International License. Read Full License 


\section{Abstract}

Global warming increases the incidence of heatstroke. After heatstroke, patients are bedridden and exhibit neurological symptoms suggesting cerebellar damage. However, the potential long-term adverse outcomes are poorly understood. We studied the cerebellum after heatstroke. In our study, motor coordination disorder significantly appeared 3 weeks post heatstroke and gradually improved to some extent over time. Demyelination was detected at 1 and 3 weeks after heatstroke at the cerebellum. Interestingly, it was not found at the corpus callosum. The Purkinje cell numbers significantly decreased at 1-, 3-, and 9-weeks post heatstroke. The intensity of synaptophysin and postsynaptic density-95 (PSD95) temporarily decreased at 3 weeks post heatstroke; however, both increased at 9 weeks post heatstroke. Motor coordination loss occurred a few weeks after heatstroke and recovered to some extent. Late-onset motor impairment was suggested to be caused by cerebellar dysfunctions that were morphologically assessed by myelin staining and immunostaining of Purkinje cells with pre-and postsynaptic markers. However, the Purkinje cell number did not recover for 9 weeks, the others, including motor coordination were partially recovered probably by synaptic reconstruction, residual Purkinje cells, and other cerebellar neuron remyelination. These phenomena were associated with late-onset neurological deficits and recovery after heatstroke.

\section{Introduction}

Heatstroke, which is a systemic disease caused by exposure to high ambient temperature (AT) and relative humidity $(\mathrm{RH})^{1}$, is estimated to increase owing to the recent global warming ${ }^{2,3}$. Since patients with heatstroke often develop multiple organ damage in critical care, they are primarily treated with whole-body cooling, hemodialysis, and plasma exchange ${ }^{4}$. Although the acute effects after a heatstroke are well-recognized, potential long-term adverse outcomes are poorly understood. Notably, the damage to the central nervous system (CNS) after a heatstroke has not been fully elucidated, despite being known to decrease the level of consciousness and function, which results in a condition similar to neuronal damage following high fevers caused by viral or bacterial infections and inflammation ${ }^{5}$. Approximately $25 \%$ of patients with heatstroke exhibit convalescent or long-term neurological deficits, including motor dysfunction and cognitive impairment.

Moreover, $>70 \%$ of patients with neurological symptoms exhibit long-term cerebellar damage, most commonly presenting with adverse long-term neurological outcomes ${ }^{6}$. The cerebellum plays an important role in motor coordination/learning, sensory integration, and coordinate transformation. Cerebellar damage results in increased postural sway, hypermetric postural responses to perturbations and optokinetic stimuli, and postural responses that are poorly coordinated with volitional movement, which induces deficits in balance and walking ${ }^{7}$. After a heatstroke, patients who exhibit certain neurological symptoms-disorientation, wobbling (Supplementary Video. S1), and vertigo-are bedridden, thereby prevented from returning to normal life, suggesting cerebellar damage. In addition to these neurological symptoms, several reports revealed abnormalities at the cerebellum upon performing head magnetic 
resonance imaging (MRI) in patients with heatstroke (Supplementary Fig. S2) ${ }^{8,9}$. Moreover, several interesting reports are showing that neurological deficits after heatstroke appeared several weeks after heatstroke rather than immediately afterward ${ }^{10,11}$ and some cases improved with time ${ }^{9}$, while others are permanent. However, it is not well understood how these late-onset neurological deficits post heatstroke appear and, in some cases, improve over time. Therefore, we focused on the effects of heatstroke on the cerebellum.

Few studies have examined the CNS using a heatstroke model. Most CNS studies performed on patients after a heatstroke have evaluated heat-induced cerebral ischemic injury ${ }^{12}$, hypothalamic injury ${ }^{13}$, and alcohol influence on the central amygdaloid nucleus ${ }^{14}$. These studies did not consider $\mathrm{RH}$, and other heatstroke studies that did not examine the CNS also disregarded the $\mathrm{RH}$ impact and created desert-like conditions $^{15-18}$. However, summer in temperate or tropical zone climates has high AT and RH. Even if the AT is the same, a higher RH accounts for a higher wet-bulb globe temperature (WBGT), suggesting a higher risk of heatstroke. Therefore, we recently established a mouse heatstroke model that accounts for AT and RH while monitoring WBGT. Our animal model presents low mortality $(<20 \%, 4$ days after heatstroke) and is considered to be suitable for evaluating long-term adverse outcomes ${ }^{19}$. In this study, we examined late onset motor coordination and cerebellar damage for 9 weeks.

\section{Results}

\section{Motor coordination disorder appeared 3 weeks post heatstroke}

Before heatstroke, the mean running time of mice in the Control (Con) and Heatstroke (HS) groups were $85.6 \pm 5.1 \mathrm{~s}$ and $84.7 \pm 5.2 \mathrm{~s}$, respectively. Two of the 36 mice in the HS group died within 1 day after heatstroke, and the remaining mice survived for 9 weeks. One week after heatstroke, the running time in the HS group was $81.8 \pm 6.8 \mathrm{~s}$, whereas the running time of the Con group was $93.1 \pm 7.0 \mathrm{~s}$. The running time in the HS group 3 weeks post heatstroke further decreased to $68.8 \pm 6.2 \mathrm{~s}$ and was significantly lower than that in the Con group $(92.7 \pm 7.7 \mathrm{~s}, P<0.05)$. The running time of the HS group gradually increased over time, whereas no differences were observed in the Con group (Fig. 1a). To eliminate feeding disorder after heatstroke, both groups of mice were weighed during the experiment, but these results were not statistically significant throughout the experiment (Fig. 1b).

\section{Demyelination was detected at 1- and 3-weeks post heatstroke in the cerebellum}

The cerebellar white matter in Con mice was dense and homogeneous to light blue (Fig. 2a, b). However, the white matter in the HS mice was heterogeneous and sponge-like (Fig. 2a, b). Semi-quantification of myelin showed that the myelin percentage was significantly decreased at 1 (Con, $70.4 \pm 2.8 \%$; HS, $59.7 \pm$ $2.8 \%, P<0.05$ ) and 3 (Con, $69.3 \pm 1.6 \%$; HS, $63.8 \pm 1.3 \%, P<0.05$ ) weeks post heatstroke (Fig. 2c), 
suggesting demyelination. However, this decrease was not statistically significant among the groups at 9 weeks, suggesting recovery.

In contrast, we compared the myelin quantity from the cerebellum with that in the corpus callosum in the same sections to determine region specificity (Fig. 2b, 2c). Interestingly, we found no significant differences between the Con and HS groups during the experiments.

\section{Purkinje cells significantly decreased after heatstroke}

The Purkinje cells in the Con mice aligned clearly in an equidistant manner (Fig. 3a). However, after heatstroke, the calbindin-positive Purkinje cells showed unequal distances and decreased numbers (Fig. $3 b)$. The Purkinje cell number in the Con vs. HS groups were counted as follows: 1-week ( $41.7 \pm 1.2$ vs. $24.0 \pm 0.4$ cells $/ \mathrm{mm}, P<0.05)$, 3 -weeks ( $41.9 \pm 0.9$ vs. $22.5 \pm 0.5$ cells $/ \mathrm{mm}, P<0.05)$, and 9 -weeks $(41.1 \pm$ 0.7 vs. $22.9 \pm 0.4$ cells $/ \mathrm{mm}, P<0.05$ ) post heatstroke, respectively (Fig. $3 \mathrm{c}$ ).

\section{The expression of PSD95 temporally decreased at 3 weeks post heatstroke}

We examined the Purkinje cell's synaptic patency using PSD95. The distribution of PSD95-positive cells was strongly localized to the axon plexus. The PSD95-positive cells in the Con group had a wide morphology like plates but had low density. The PSD95-positive cells 1 week after heatstroke were localized to the axon hillock of the Purkinje cells (looked like pen points). After 3 weeks, the immunoreactions decreased, although the calbindin-positive Purkinje cells were stained in the region. The positive reactions reappeared more heterogeneously at 9 weeks post heatstroke. Moreover, PSD 95 immunoreactivity was again observed around the axon hillock of some residual Purkinje cells and appeared to have stronger intensities than observed in the Con group (Fig. 4). The co-staining of PSD95 with synaptophysin revealed that the intensity of synaptophysin immunoreactivity around the Purkinje cells was weakest at 3 weeks post heatstroke; however, both increased at 9 weeks post heatstroke (Fig. $5)$.

\section{Discussion}

It is estimated that recent global warming will cause an increase in patients with heatstroke in the near future. However, there is less understanding of potential long-term adverse outcomes, although patients often exhibit neurological deficits ${ }^{8,20}$.

In this study, we evaluated the neurological deficits using the rotarod test and brain histological evaluations (in the cerebellum) for 9 weeks using our heatstroke model, because disappearing and swelling of the Purkinje cells have been reported in autopsied brain tissues of patients with heatstroke ${ }^{21}$. Observation of brain diffusion-weight MRI revealed hyperintensity in the bilateral cerebellar medullas in patients with heatstroke ${ }^{22}$. These reports indicate that Purkinje cell protection is a key therapeutic target in patients with heatstroke. 
In our study, the running time was significantly shorter at 3 weeks post heatstroke and gradually returned to baseline at 9 weeks post heatstroke. These results suggest motor function/coordination impairment in our heatstroke model. In patients with multiple sclerosis, cerebellar ataxia commonly occurs; cerebellar neuronal degeneration has been reported ${ }^{23}$. Therefore, we examined the morphology of the brain by KB staining, but we could not recognize apparent cerebral infarction or hemorrhage. However, the cerebellum showed decreased intensity with KB stain and was recognized as sponge-like in the HS group. Semiquantification of the KB-stained region revealed that the cerebellar white matter was significantly demyelinated between 1- and 3-weeks post heatstroke in the HS group. However, the intensity did not significantly differ from that of the Con group at 9 weeks post heatstroke, suggesting remyelination. In contrast, demyelination could not be recognized in the corpus callosum during the experiments; the demyelination was cerebellar-specific and might not have recovered until 9 weeks.

The calbindin-positive Purkinje cells in the Con mice were aligned clearly on the Purkinje cell layer equidistantly. The cell numbers had significantly decreased at 1 week post heatstroke and did not recover during the experimental periods, suggesting that the Purkinje cells degenerated. These results, including the rotarod test, suggested that demyelination after heatstroke-induced cerebellar ataxia resulted in impaired motor coordination. These results resemble the outcome seen in human patients with heatstroke $e^{8,20-22}$.

However, myelin recovered 9 weeks post heatstroke along with the motor coordination, although the number of Purkinje cells did not recover during the experimental periods. To explain this discrepancy, we immunostained for PSD95 and synaptophysin to examine synaptic patency and observed the Purkinje layer at a higher magnification. The PSD 95 was highly concentrated and tightly bound to the postsynaptic density of type 1 glutamatergic synapses, suggesting its critical role in protein assembly, synaptic development, and neural plasticity ${ }^{23-25}$. Synaptophysin is localized to the presynaptic vesicle membrane and contributes to membrane transport ${ }^{26}$. Its reduction has been considered to correlate with synaptic and neural impairments in Alzheimer's disease and traumatic brain injury ${ }^{27-30}$. The PSD95 immunoreactions were recognized in the same direction of the axon plexus surrounding the axon hillock of each Purkinje cell as previously reported ${ }^{31}$ and distributed widely like plates or sheets along with the Purkinje cells. The degenerated Purkinje cells could not recover, and the cerebellar neuronal connections were temporally disrupted after heatstroke. However, the synaptic patency and myelin staining results, including motor coordination, suggest that the surviving and residual Purkinje cells and the other cerebellar neurons were remyelinated and reconstructed new synaptic connections, probably inducing the recovery of partial motor coordination. It was suggested that these phenomena were associated with lateonset neurological deficits and recovery after heatstroke.

Cerebellum has diversity and dynamism ${ }^{32}$ and cerebellar dysfunction improved to some extent with time in our rodent model of heatstroke. Thought, there is a case that showed permanent neurological deficit in human heatstroke. Synaptic impairment might not be improved if the Purkinje cell damages were severe after heatstroke. Our results indicated that there is a time before demyelination or synaptic impairment. 
Therefore, some therapeutic intervention during this period may contribute to improve neurological deficit after heatstroke.

In this study, we neither analyze skeletal muscle impairment nor determine the spinal cord impairment and the peripheral nerve fiber innervation because of a circulatory failure due to massive hemorrhagic shock and ischemia-induced motor neuron necrosis of the spinal cord's ventral horn ${ }^{33}$. Moreover, we have not systematically determined diffuse cerebral ischemia. Further analysis is needed to clarify the heat exposure (HE) pathogeny and progression.

\section{Conclusions}

In the present study, we determined the motor coordination loss within a few weeks after heatstroke onset. Motor impairment was suggested to be caused by cerebellar dysfunctions that were morphologically assessed by myelin staining and immunostaining of Purkinje cells with pre-and postsynaptic markers. However, although the Purkinje cell number did not recover for 9 weeks, the others, including motor coordination, were partially recovered, probably by synaptic reconstruction, residual Purkinje cell, and other cerebellar neuron remyelination. It was suggested that these phenomena were associated with late-onset neurological deficits and recovery after heatstroke. Further research is required to clarify the mechanism of cerebellar dysfunction in heatstroke to establish new therapeutic strategies.

\section{Methods}

Male C57/BL6J mice (aged 10 weeks) were used in this study. All animals were purchased from SLC Japan Inc. (Shizuoka, Japan). The mice were allowed free access to food and water and were maintained on a $12 \mathrm{~h}$ light/dark cycle at room temperature $\left(24 \pm 2^{\circ} \mathrm{C}\right)$ with constant humidity $(40 \pm 15 \%)$. All experimental procedures involving animals and clinical data were approved and overseen by the Institutional Animal Care and Use Committee of Showa University (\#09022, 02003), which adhered to the ARRIVE guidelines. Video of wobbling after human heat stroke and head magnetic resonance imaging (MRI) in patients with heatstroke were obtained from participants. All experimental protocols were approved and overseen by Clinical Trial Review Board of Showa University (F2019C83) which adhered to the CIOMS Ethical Guidelines for Biomedical Research. Informed consent was obtained from all participants and/or their legal guardian. Research have been performed in accordance with the Declaration of Helsinki.

\section{Protocol of heatstroke}

The high AT and $\mathrm{RH}$, resembling temperate/tropical summers, heatstroke model was based on our previous study ${ }^{19}$. A semi-enclosed heatstroke chamber $(200 \times 340 \times 300 \mathrm{~mm})$ made of acrylic was created by vertically stacking the animal cages in a greenhouse-like construction. An ultrasonic humidifier (USB68, Sanwa, Japan) and a digital thermo-hygrometer (AD-5696; CA\&D Company, Japan) were used for the humidification and monitoring of AT, RH, and WBGT. The heatstroke chamber was placed in an incubator 
(Bio-chamber, BCP-120F; TITEC, Japan) and preheated to the desired experimental temperature for $\geq 3 \mathrm{~h}$. The humidifier was started $3 \mathrm{~h}$ before heatstroke to create a hot and humid environment. Meanwhile, the mice were given $3 \mathrm{~h}$ of water restriction, and the mildly dehydrated mice were placed in the heatstroke chamber and exposed to high AT $\left(41^{\circ} \mathrm{C}\right)$ and $\mathrm{RH}(>99.0 \%)$ for $60 \mathrm{~min}$. Subsequently, they were returned to the animal cage, where they could access food and water. No heat-exposed animals were used as controls (Con group).

\section{Behavioral study (Rotarod test)}

The rotarod test was performed according to previous reports ${ }^{34}$. A rotarod treadmill (Muromachi Kikai, Japan) consisted of a plastic rod (diameter, $3 \mathrm{~cm}$; length, $10 \mathrm{~cm}$ ) flanked by four large round plates (diameter, $57 \mathrm{~cm}$ ). The rod rotates at a constant speed of $4 \mathrm{rpm}$ at the beginning and continuously accelerates to a speed of $40 \mathrm{rpm}$ for $5 \mathrm{~min}$. The time each mouse spent on the rod was measured. Mice were trained with rotarod once a week for five times before heatstroke. The behavior test in a mouse was performed twice within an interval of 5 min each and was expressed as the average of the trials. The animals were divided into two groups (HS and Con) according to the scores of the last training ( $n=36$ in each group). The HS group mice were subjected to heat for an hour. The Con group (Without HE) mice were prepared as a control. The HS and Con groups were subjected to behavioral tests at 1-, 3-, 5-, 7-, and 9-weeks post heatstroke (Supplementary Fig. S3a).

\section{Tissue preparation}

Under sodium pentobarbital ( $50 \mathrm{mg} / \mathrm{kg}$, i.p.) anesthesia, the mice (HS, $\mathrm{n}=9$, per time course in each group) at 1-, 3-, and 9-weeks post heatstroke were transcardially perfused with $0.9 \% \mathrm{NaCl}$, followed by $10 \%$ neutralized formalin. The brain was removed and divided into two parts along the longitudinal cerebral fissure. Paraffin-embedded specimens of the right hemisphere of the brain were prepared. Then, eight sagittal sections were sliced at a thickness of $5 \mu \mathrm{m}$ at every $200 \mu \mathrm{m}$ interval from the cerebral longitudinal fissure for histological examination, as described below. Age-matched Con group mice were prepared to eliminate the influence of senescence (Supplementary Fig. S3b).

\section{Semi-quantification of cerebellar white matter demyelination}

Cerebellar white matter demyelination was examined with Klüver-Barrera (KB) method without Nissl staining $^{33,35}$. A series of eight sagittal sections, as aforementioned, were observed. The Luxol fast blue intensity, which stains myelin (light blue) in the white matter, was then semi-quantified to evaluate demyelination. These areas of $>70 \mu \mathrm{m}^{2}$ were manually traced and converted to black and white; black areas were semi-quantified using Scion Image for Windows (Scion Corporation, USA). The pixels of the black myelinated regions were divided by the pixels from the total traced areas and expressed as percentages. Eight serial sections were averaged for each animal. These procedures were performed by investigators (H.Y. and K.Y.) who were blinded to the experimental groups. 
Another series of eight sagittal sections were immunostained with antibodies against calbindin D-28k (Calbindin) and used for Purkinje cells counting ${ }^{36}$. After removing the paraffin using a series of xylene/alcohol solutions, the sections were incubated in $10 \mathrm{mM}$ sodium citrate buffer $(\mathrm{pH}=5.0)$ for 25 min for heat-induced antigen retrieval and immersed in $0.3 \% \mathrm{H}_{2} \mathrm{O}_{2}$ /methanol for 30 min to block the endogenous peroxidase reaction. The sections were then incubated with mouse Ig blocking reagent (M.O.M. ; Vector, USA), followed by $5 \%$ goat serum wash to diminish the mouse endogenous immunoglobulin and non-specific reactions. The sections were then incubated overnight with a monoclonal mouse anti-calbindin antibody (1:2000; Swant, Switzerland) and incubated for 90 min with a biotinylated goat anti-mouse $\lg G$ the next day (1:200, DAKO, CA). The immunoreactions were visualized by incubating with an avidin-biotin complex solution (Vector, USA) and diaminobenzidine (Sigma, USA). The calbindin-immunopositive Purkinje cells were determined and counted using CellSens Standard software (Olympus, Japan). The number of Purkinje cells in the molecular layer of the cerebellum more than $2 \mathrm{~mm}$ in length was counted twice manually in different lobes of the same section, and the average was calculated. Cell counting was repeated in the eight sagittal sections. This was also performed by an investigator (HY or KY) who were blinded to the experimental groups.

\section{Immunostaining of synaptic markers}

Post- and presynaptic markers were co-stained in the cerebellum at 1, 3, and 9 weeks after heatstroke to estimate the Purkinje cell's synaptic patency. After heat-induced antigen retrieval and blocking according to the protocol as mentioned above, the sections were incubated with either monoclonal rabbit anticalbindin antibody (1:100, Cell Signaling, USA) or polyclonal rabbit anti-synaptophysin antibody (1:200, Proteintech, USA), with monoclonal mouse anti-postsynaptic density 95 (PSD95) antibody (1:400, BD, USA) overnight at $4^{\circ} \mathrm{C}$. After washing, the sections were incubated with Alexa488-conjugated goat antirabbit IgG antibody (1:400; Thermo Fisher Scientific, USA) and Alexa 546-conjugated goat anti-mouse IgG antibody (1:400; Invitrogen, USA). Subsequently, cell nuclei were stained with 4,6-diamidine-2phenylindole dihydrochloride (1:10,000; Roche, Germany) and incubated in $1.0 \mathrm{mM} \mathrm{CuSO}_{4}$ in $50 \mathrm{mM}$ ammonium acetate buffer $(\mathrm{pH}=5.0)$ to diminish autofluorescence ${ }^{37,38}$. Fluorescence was detected using an Axio Imager optical sectioning microscope with ApoTome II (Carl Zeiss, Germany). For control staining, the same steps were performed except for incubation with primary antibodies.

\section{Statistical analysis}

Data are reported as mean \pm standard error of the mean. The student's t-test was used for comparisons between the two groups. Statistical significance was set at $P<0.05$.

\section{Declarations}

Acknowledgments: We would like to thank Editage (www.editage.com) for their English language editing.

Authors' contributions: K.M. designed and performed this research. M.N. also performed this research and contributed equally to this article. H.O. contributed to the immunostaining of tissues. K.H. advised the 
histopathological findings as a clinical pathologist. K.D. supervised the contents. All authors reviewed the manuscript.

Data availability statement: Not applicable

Competing interests: Authors declare no conflicts of interest.

Ethics declarations: All experimental procedures involving animals were approved and overseen by the Institutional Animal Care and Use Committee of Showa University, which adhered to the ARRIVE guidelines. All human research protocols were approved and overseen by Clinical Trial Review Board of Showa University which adhered to the CIOMS Ethical Guidelines for Biomedical Research. Informed consent was obtained from all participants and/or their legal guardian. Research have been performed in accordance with the Declaration of Helsinki.

Approval for animal experiments: All experimental procedures involving animals and clinical data were approved and overseen by the Institutional Animal Care and Use Committee of Showa University (\#09022, 02003), which adhered to the ARRIVE guidelines.

Approval for human experiments: All human research protocols were approved and overseen by Clinical Trial Review Board of Showa University (F2019C83) which adhered to the CIOMS Ethical Guidelines for Biomedical Research. Informed consent was obtained from all participants and/or their legal guardian. Research have been performed in accordance with the Declaration of Helsinki.

Funding: This work was supported by JSPS KAKENHI Grant Numbers 19 K09442 (K.M.), 19 K22779 (K.D.), and $21 \mathrm{H} 03036$ (K.D.).

\section{References}

1. Epstein, Y. \& Yanovich, R. Heatstroke. N. Engl. J. Med. 380, 2449-2459, 10.1056/NEJMra1810762 (2019).

2. Meehl, G. A. \& Tebaldi, C. More intense, more frequent, and longer lasting heat waves in the $21 \mathrm{st}$ century. Science 305, 994-997, 10.1126/science.1098704 (2004).

3. Sherwood, S. C. \& Huber, M. An adaptability limit to climate change due to heat stress. Proc. Natl Acad. Sci. U. S. A. 107, 9552-9555, 10.1073/pnas.0913352107 (2010).

4. Hifumi, T., Kondo, Y., Shimizu, K. \& Miyake, Y. Heat stroke. J. Intensive Care 6, 30,:10.1186/s40560018-0298-4 (2018).

5. Harden, L. M., Kent, S., Pittman, Q. J. \& Roth, J. Fever and sickness behavior: friend or foe? Brain Behav. Immun. 50, 322-333, 10.1016/j.bbi.2015.07.012 (2015).

6. Lawton, E. M., Pearce, H. \& Gabb, G. M. Review article: environmental heatstroke and long-term clinical neurological outcomes: A literature review of case reports and case series 2000-2016. Emerg. Med. Australas. 31, 163-173, 10.1111/1742-6723.12990 (2019). 
7. Marsden, J. F. Cerebellar ataxia. Handb. Clin. Neurol. 159, 261-281, 10.1016/B978-0-444-63916$5.00017-3$ (2018).

8. Li, J. et al. Diffusion tensor imaging of the cerebellum in patients after heat stroke. Acta Neurol. Belg. $115,147-150,10.1007 /$ s13760-014-0343-6 (2015).

9. De Cori, S. et al. Clinical recovery despite cortical cerebral and cerebellar damage in heat stroke. Neuroradiol. J. 23, 35-37, 10.1177/197140091002300105 (2010).

10. Jung, I., Choi, S. Y., Kim, H. J. \& Kim, J. S. Delayed vestibulopathy after heat exposure. J. Neurol. 264, 49-53,:10.1007/s00415-016-8322-x (2017).

11. Lo, Y. C., Yen, D. H., Guo, W. Y. \& Yang, C. C. Diffuse cerebral cortex, cerebellar cortex and basal ganglia injury: a rare MR imaging manifestation of heat stroke. Neuroradiol. J. 20,37-40, 10.1177/197140090702000105 (2007).

12. Lin, M. T. Heatstroke-induced cerebral ischemia and neuronal damage. Involvement of cytokines and monoamines. Ann. N. Y. Acad. Sci. 813, 572-580, 10.1111/j.1749-6632.1997.tb51748.x (1997).

13. Lee, K. L., Niu, K. C., Lin, M. T. \& Niu, C. S. Attenuating brain inflammation, ischemia, and oxidative damage by hyperbaric oxygen in diabetic rats after heat stroke. J. Formos. Med. Assoc. 112, 454462, 10.1016/j.jfma.2012.02.017 (2013).

14. Kibayashi, K., Nakao, K. \& Shojo, H. Hyperthermia combined with ethanol administration induces cfos expression in the central amygdaloid nucleus of the mouse brain. A possible mechanism of heatstroke under the influence of ethanol intake. Int. J. Legal Med. 123, 371-379, 10.1007/s00414008-0278-7 (2009).

15. Shen, K. H., Lin, C. H., Chang, H. K., Chen, W. C. \& Chen, S. H. Premarin can act via estrogen receptors to rescue mice from heatstroke-induced lethality. Shock 30, 668-674, 10.1097/SHK.0b013e31817538cb (2008).

16. Bouchama, A. et al. Inflammatory, hemostatic, and clinical changes in a baboon experimental model for heatstroke. J. Appl. Physiol. 98, 697-705, 10.1152/japplphysiol.00461.2004 (2005).

17. Leon, L. R., DuBose, D. A. \& Mason, C. W. Heat stress induces a biphasic thermoregulatory response in mice. Am. J. Physiol. Regul. Integr. Comp. Physiol. 288, R197-204, 10.1152/ajpregu.00046.2004 (2005).

18. Chen, C. M. et al. Activated protein C therapy in a rat heat stroke model. Crit. Care Med. 34, 19601966, 10.1097/01.CCM.0000224231.01533.B1 (2006).

19. Miyamoto, K. et al. A novel mouse model of heatstroke accounting for ambient temperature and relative humidity. J. Intensive Care 9, 35, 10.1186/s40560-021-00546-8 (2021).

20. Albukrek, D., Bakon, M., Moran, D. S., Faibel, M. \& Epstein, Y. Heat-stroke-induced cerebellar atrophy: clinical course, CT and MRI findings. Neuroradiology 39, 195-197, 10.1007/s002340050392 (1997).

21. Malamud, N., Haymaker, W. \& Custer, R. P. Heat stroke; a clinico-pathologic study of 125 fatal cases. Mil. Surg. 99, 397-449 (1946). 
22. Hirayama, I., Inokuchi, R., Ueda, Y. \& Doi, K. Heat stroke lesions in the globus pallidus. Intern. Med. 59, 1015-1016, 10.2169/internalmedicine.3317-19 (2020).

23. Redondo, J. et al. Purkinje cell pathology and loss in multiple sclerosis cerebellum. Brain Pathol. 25, 692-700, 10.1111/bpa.12230 (2015).

24. Kim, E., Cho, K. O., Rothschild, A. \& Sheng, M. Heteromultimerization and NMDA receptor-clustering activity of Chapsyn-110, a member of the PSD-95 family of proteins. Neuron 17, 103-113, 10.1016/s0896-6273(00)80284-6 (1996).

25. Sheng, M. \& Hoogenraad, C. C. The postsynaptic architecture of excitatory synapses: a more quantitative view. Annu. Rev. Biochem. 76, 823-847, 10.1146/annurev.biochem.76.060805.160029 (2007).

26. Kwon, S. E. \& Chapman, E. R. Synaptophysin regulates the kinetics of synaptic vesicle endocytosis in central neurons. Neuron 70, 847-854, 10.1016/j.neuron.2011.04.001 (2011).

27. Reiss, A. B., Arain, H. A., Stecker, M. M., Siegart, N. M. \& Kasselman, L. J. Amyloid toxicity in Alzheimer's disease. Rev. Neurosci. 29, 613-627, 10.1515/revneuro-2017-0063 (2018).

28. Lecca, D. et al. (-)-Phenserine and the prevention of pre-programmed cell death and neuroinflammation in mild traumatic brain injury and Alzheimer's disease challenged mice. Neurobiol. Dis. 130, 104528, 10.1016/j.nbd.2019.104528 (2019).

29. Aggarwal, S. et al. Relationship of cerebral blood flow and cerebral swelling to outcome in patients with acute fulminant hepatic failure. Transplant. Proc. 23, 1978-1979 (1991).

30. Manek, R. et al. Protein biomarkers and neuroproteomics characterization of microvesicles/exosomes from human cerebrospinal fluid following traumatic brain injury. Mol. Neurobiol. 55, 6112-6128, 10.1007/s12035-017-0821-y (2018).

31. Hunt, C. A., Schenker, L. J. \& Kennedy, M. B. PSD-95 is associated with the postsynaptic density and not with the presynaptic membrane at forebrain synapses. J. Neurosci. 16, 1380-1388, 10.1523/JNEUROSCI.16-04-01380.1996. (1996)

32. De Zeeuw, C. I., Lisberger, S. G. \& Raymond, J. L. Diversity and dynamism in the cerebellum. Nat. Neurosci. 24, 160-167, 10.1038/s41593-020-00754-9 (2021).

33. Kudo, Y. et al. Neuronal damage in rat brain and spinal cord after cardiac arrest and massive hemorrhagic shock. Crit. Care Med. 34, 2820-2826, 10.1097/01.CCM.0000242522.48734.64 (2006).

34. Matsuura, S., Shuvaev, A. N., lizuka, A., Nakamura, K. \& Hirai, H. Mesenchymal stem cells ameliorate cerebellar pathology in a mouse model of spinocerebellar ataxia type 1 . Cerebellum 13, 323-330, 10.1007/s12311-013-0536-1 (2014).

35. Wakita, H. et al. Axonal damage and demyelination in the white matter after chronic cerebral hypoperfusion in the rat. Brain Res. 924, 63-70, 10.1016/s0006-8993(01)03223-1 (2002).

36. Girard, F., Venail, J., Schwaller, B. \& Celio, M. R. The EF-hand Ca(2+)-binding protein super-family: a genome-wide analysis of gene expression patterns in the adult mouse brain. Neuroscience 294, 116$155,10.1016 /$ j.neuroscience.2015.02.018 (2015). 
37. Schnell, S. A., Staines, W. A. \& Wessendorf, M. W. Reduction of lipofuscin-like autofluorescence in fluorescently labeled tissue. J. Histochem. Cytochem. 47, 719-730, 10.1177/002215549904700601 (1999).

38. Hiraiwa, A. \& Milner, E. C. Locus-specific vector/primer systems for rapid cloning of allelic variants. Gene 71, 193-199, 10.1016/0378-1119(88)90091-1 (1988)

\section{Figures}

\section{Figure 1}

\section{Motor coordination and body weight for 9 weeks after heatstroke}

Running time by the rotarod test (a) and body weight (b, BW) in the control (Con) and heatstroke (HS) animals. The running time in the HS animals 3 weeks post heatstroke significantly decreased as compared to that in Con animals. BW in the HS and Con group did not change statistically over the experiments. Data are expressed as the mean \pm standard deviation (SD). ( ${ }^{*} P<0.05$, Student's t-test).

\section{Figure 2}

\section{Cerebellum myelin sheaths in heatstroke animals temporarily decreased with Klüver-Barrera staining} after heatstroke

Myelin sheaths were stained light blue with Klüver-Barrera stain. The amount of myelination was semiquantified in the white matter (WM) of the cerebellum and corpus callosum (CC) after heatstroke. (a) Schematic low-magnification images of the cerebellum and telencephalon indicate obvious light blue staining of myelin sheaths. (b) Representative higher magnification images of WM and CC. The WM of the cerebellum in the control (Con) mice was dense and homogeneous. However, those in the heatstroke (HS) mice after heatstroke were heterogeneous and sponge-like, although these were not seen in the CC. (c) Semi-quantification of the myelin staining revealed that the myelinated percentages were significantly decreased at 1- and 3-weeks post heatstroke, and it recovered 9 weeks later. No differences were observed between the HS and Con groups in the CC. Data are expressed as the mean $\pm \mathrm{SD}$. ( ${ }^{\star} P<0.05$, Student's ttest). ML, molecular layer; PL, Purkinje cell layer; GL, granular cell layer; WM, white matter; CTX, cerebral cortex; CC, corpus callosum; HIP, hippocampus; SD, Standard deviation

\section{Figure 3}




\section{Calbindin-positive Purkinje cells were decreased after heatstroke.}

To assess the Purkinje cells, calbindin was immunostained in the brain and the calbindin-positive Purkinje cell numbers were counted. (a) Representative image of calbindin-immunoreactions in the cerebellum. Calbindin-immunoreactions stained the soma of Purkinje cells, the dendritic fibers that were widely stained in the molecular layer (ML), and the white matter. (b) Higher magnification images of the Purkinje cell layers in the control (Con; left) and 1 to 9 weeks after heatstroke. Purkinje cells in Con animals were aligned in an equidistant manner. However, the number of cells decreased after heatstroke. (c) The Purkinje cell numbers of HS group were significantly decreased during the experimental periods after heatstroke $(1,3$, and 9 weeks, $n=9$, respectively) compared with the Con group $(n=9)$. Data are expressed as the mean \pm SD. ( ${ }^{\star} P<0.05$, Student's t-test).

ML, molecular layer; PL, Purkinje cell layer; GL, granular cell layer; WM, white matter; SD, standard deviation

\section{Figure 4}

\section{The immunostaining of postsynaptic density 95 in the Purkinje cells after heatstroke}

(a) Representative images of anti-postsynaptic density 95 (PSD95) (red), a postsynaptic marker, and calbindin (green) immunoreactions in the cerebellum. The PSD95 immunoreactions were observed in the Purkinje cells. Blue is 4',6-diamidino-2-phenylindole nucleic staining. (b) The higher magnified images indicated that the PSD95-immunoreactions were located in the axon hillock of each Purkinje cell. The immunoreactions in the control group were recognized widely like plates with relatively lower density in the Purkinje cells (arrowhead). The PSD95 immunoreactions 1 week after heatstroke (arrow) were gathered and concentrated to the axon hillock of the Purkinje cells like pen points. The immunoreactions were decreased at 3 weeks and reappeared more heterogeneously at 9 weeks.

ML, molecular layer; PL, Purkinje cell layer; GL, granular cell layer; WM, white matter

\section{Figure 5}

\section{The expression of postsynaptic density 95 and synaptophysin around Purkinje cells}

To estimate synaptic connections, pre- (synaptophysin, green) and post- (PSD95, red) synaptic markers were co-stained in the cerebellum and were observed with higher magnification images. Both postsynaptic density 95 and synaptophysin immunoreactions in the Purkinje cell layer decreased 3 weeks after heatstroke and increased again after 9 weeks. 


\section{Supplementary Files}

This is a list of supplementary files associated with this preprint. Click to download.

- SupplementaryFig.S2.tif

- SupplementaryFig.S3.tif

- SupplementaryInformation20220221.docx

- Supplementaryvideo.S1.mp4 